\title{
Developments in finite Phan theory
}

\author{
Ralf Gramlich* \\ Keywords : building, twin building, algebraic group, presentation, amalgam, Curtis-Tits \\ presentation, Phan's presentation, Solomon-Tits theorem \\ MSC 2000: 05-06, 05E25, 20-06, 20D05, 20D06, 51-06, 51A50, 51B25, 51E24, 57-06, \\ $57 \mathrm{Q} 10$
}

\section{Introduction}

Geometric methods in the theory of Chevalley groups and their generalisations have made tremendous advances during the last few decades. Among the most noteworthy and influential of these advances are the systematic application of the concept of amalgams based on [49, 59, 117], the local-to-global approach [136], ingenious applications of combinatorial topology and geometric group theory (as in $[2,91,92,102,137]$ ), the theory of abstract root groups [127, $129,130]$, and the interaction of Kac-Moody groups and twin buildings [34, $35,36,39,38,138,140]$. These methods have proven fruitful over and over again in proving, simplifying and generalising several results in group theory and have had their impact in other areas of mathematics.

The present survey attempts to give a report on the results and on the developments in recent years and to serve as a guide to the literature for the project called Curtis-Phan-Tits Theory (or, short, Phan Theory). This project has been initiated in [19] with the goal to revise Phan's results [106, 107] on presentations of twisted forms of finite Chevalley groups via rank one and rank two groups in order to make them accessible for the ongoing revision of the classification of the finite simple groups $[60,61,62,63,64,65]$.

The main impact of Phan's results $[106,107]$ in the classification can be seen in [14] side by side with the famous Curtis-Tits Theorem established in [45, 134], [135, Theorem 13.32]; see also [62, Section 2.9] and Section 4 of this survey. As in this survey my main concern is the revision of Phan's results,

\footnotetext{
${ }^{*}$ The author gratefully acknowledges his Heisenberg fellowship of the Deutsche Forschungsgemeinschaft.
} 




\subsubsection{Homotopy}

The following three operations are called elementary homotopies of paths: substituting a subsequence $x, x$ (a repetition) by $x$, or vice versa, substituting a subsequence $x, y, x$ (a return) by $x$, or vice versa, or substituting a subsequence $x, y, z, x$ (a triangle) by $x$ or vice versa, provided that $x, y, z$ form a simplex. Two paths are homotopically equivalent if they can be obtained from one another in a finite sequence of elementary homotopies. A cycle, that is, a path with $x_{0}=x_{n}$, is called null-homotopic, if it is homotopically equivalent to the trivial path $x_{0}$. The fundamental group $\pi_{1}(\mathcal{S}, x)$, where $x \in X$, is the set of homotopy classes of cycles based at $x$ where the product is defined to be concatenation of cycles. The fundamental group is independent of the choice of the base vertex $x$ inside a fixed connected component, while it may vary for base vertices in distinct connected components. When considering connected complexes only, the coverings of $\mathcal{S}$, taken up to a certain natural equivalence, correspond bijectively to the subgroups of $\pi_{1}(\mathcal{S}, x)$, cf. [116, $\left.\S 55\right]$. A connected complex $\mathcal{S}$ is called simply connected, if each covering $\mathcal{S}^{\prime} \rightarrow \mathcal{S}$ with connected $\mathcal{S}^{\prime}$ is an isomorphism, or equivalently $([116, \S 56],[120$, Section 2.5$])$, if $\pi_{1}(\mathcal{S}, x)=1$.

\subsubsection{Flag complexes and realisations}

With every pregeometry $\mathcal{G}=(X$, typ,$*)$ one can associate its flag complex which is a simplicial complex defined on the set $X$ whose simplices are the flags of $\mathcal{G}$. The flag complex of a pregeometry $\mathcal{G}$ is pure if and only if $\mathcal{G}$ is a geometry. A pregeometry $\mathcal{G}$ is simply connected, if such is its flag complex.

For a simplicial complex $\mathcal{S}=(X, \Delta)$ denote by $|\mathcal{S}|$ the set of all functions $\alpha$ from $X$ to the real unit interval $I$ satisfying that the set $\{v \in X \mid \alpha(v) \neq 0\}$ is contained in $\Delta$ and that $\sum_{v \in X} \alpha(v)=1$, i.e., $|\mathcal{S}|$ is obtained from $\mathcal{S}$ via barycentric coordinates. In this survey I consider the weak (coherent) topology on $|\mathcal{S}|$, cf. [120, 3.1.14], and call it the realisation of $\mathcal{S}$. With respect to this topology, the fundamental group $\pi_{1}(\mathcal{S}, x)$ defined combinatorially in Section 2.2.2 coincides with the usual fundamental group defined topologically, see [120, 3.6.17].

\subsubsection{Wedges of spheres}

Let $X$ and $Y$ be pointed spaces, i.e., topological spaces with distinguished base points $x_{0}$ and $y_{0}$. Then the wedge sum $X \vee Y$ of $X$ and $Y$ is the quotient of the disjoint union $X \sqcup Y$ by the identification $x_{0} \sim y_{0}$, i.e.,

$$
X \vee Y:=(X \sqcup Y) /\left\{x_{0} \sim y_{0}\right\} .
$$








\subsubsection{Shapes}

For a maximal flag $F$ a shape is a subset $\mathcal{W}$ of $2^{F}$ such that $2^{F} \ni U^{\prime} \supset U \in \mathcal{W}$ implies $U^{\prime} \in \mathcal{W}$, i.e., $\mathcal{W}$ is a subset of the power set of $F$ that is closed under passing to supersets. The amalgam of shape $\mathcal{W}$ with respect to $\mathcal{G}, G, F$ is the family $\left(G_{U}\right)_{U \in \mathcal{W}}$, where $G_{U}$ is the stabiliser of $U \in \mathcal{W}$ in $G$. It is denoted by $\mathcal{A}_{\mathcal{W}}(\mathcal{G}, G, F)$. Shapes allow for a neat explanation why many presentations of groups based on amalgams of parabolics are redundant.

Redundancy Theorem ([78, Theorem 3.3]). Let $\mathcal{G}=(X, *$, typ) be a geometry over some finite set $I$, let $G$ be a flag-transitive group of automorphisms of $\mathcal{G}$, and let $F$ be a maximal flag of $\mathcal{G}$. Moreover, let $\mathcal{W} \subseteq 2^{F}$ be a shape, assume that for each flag $U \in 2^{F} \backslash \mathcal{W}$ the residue $\mathcal{G}_{U}$ is simply connected, and let $\mathcal{A}(\mathcal{G}, G, F)$ and $\mathcal{A}_{\mathcal{W}}(\mathcal{G}, G, F)$ be the amalgam of maximal parabolics, resp. the amalgam of shape $\mathcal{W}$ of $\mathcal{G}$ with respect to $G$ and $F$. Then $G=\mathcal{U}\left(\mathcal{A}_{\mathcal{W}}(\mathcal{G}, G, F)\right)$ and, if $\emptyset \notin \mathcal{W}$, furthermore $G=\mathcal{U}(\mathcal{A}(\mathcal{G}, G, F))=\mathcal{U}\left(\mathcal{A}_{\mathcal{W}}(\mathcal{G}, G, F)\right)$.

\section{Phan's Theorems}

\subsection{Phan's first theorem}

The first of the group-theoretic identification theorems I discuss in this survey is Phan's first theorem. In 1977 Kok-Wee Phan [106] — the namesake of the theory reported on in this survey- described a method of identification of a group $G$ as a quotient of the unitary group $\mathrm{SU}_{n+1}\left(q^{2}\right)$ via a generating configuration consisting of subgroups $\mathrm{SU}_{2}\left(q^{2}\right)$ and $\mathrm{SU}_{3}\left(q^{2}\right)$ and $\mathrm{SU}_{2}\left(q^{2}\right) \times \mathrm{SU}_{2}\left(q^{2}\right)$ in $G$.

\subsubsection{Phan systems}

It is helpful to begin by looking at this configuration of subgroups inside the group $\mathrm{SU}_{n+1}\left(q^{2}\right)$ in order to motivate the forthcoming definitions. For $n \geq 2$ and $q$ a prime power, consider $G=\mathrm{SU}_{n+1}\left(q^{2}\right)$ acting as a matrix group with respect to an orthonormal basis on a unitary $(n+1)$-dimensional vector space over $\mathbb{F}_{q^{2}}$, and let $U_{i} \cong \mathrm{SU}_{2}\left(q^{2}\right), i=1,2, \ldots, n$, be the subgroups of $G$ corresponding to the $(2 \times 2)$-blocks along the main diagonal represented as matrix groups with respect to the chosen orthonormal basis. Let $T_{i}$ be the diagonal subgroup in $U_{i}$ with respect to this basis, which is a maximal torus of $U_{i}$ of size $q+1$. For $q \geq 3$ and $1 \leq i, j \leq n$ the subgroups $U_{i}$ and $T_{i}$ satisfy the following axioms:

(P1) if $|i-j|>1$, then $[x, y]=1$ for all $x \in U_{i}$ and $y \in U_{j}$, 



$$
\text { (wP3) } G=\left\langle U_{i, j} \mid 1 \leq i<j \leq n\right\rangle \text {. }
$$

Here a standard pair in the matrix group $\mathrm{SU}_{3}\left(q^{2}\right)$ is a pair of subgroups isomorphic to $\mathrm{SU}_{2}\left(q^{2}\right)$ conjugate as a pair to the two block-diagonal groups isomorphic to $\mathrm{SU}_{2}\left(q^{2}\right)$, i.e., these two groups centralise a pair of orthonormal vectors of the natural module of $\mathrm{SU}_{3}\left(q^{2}\right)$. Standard pairs in central quotients of $\mathrm{SU}_{3}\left(q^{2}\right)$ are defined as the images under the canonical homomorphism of standard pairs of $\mathrm{SU}_{3}\left(q^{2}\right)$.

full screen

close quit

ACADEMIA PRESS

N

$\widehat{\underline{\text { IIIIII }}}$ UNIVERSITEIT GENT

\subsubsection{Non-degenerate unitary space}

Consider $G \cong \mathrm{SU}_{n+1}\left(q^{2}\right)$ as a matrix group with respect to an orthonormal basis of its natural module and let $\mathcal{A}$ be the amalgam consisting of the blockdiagonal subgroups $\mathrm{SU}_{2}\left(q^{2}\right)$ and $\mathrm{SU}_{3}\left(q^{2}\right)$ and $\mathrm{SU}_{2}\left(q^{2}\right) \times \mathrm{SU}_{2}\left(q^{2}\right)$. One has to prove that the universal enveloping group of the amalgam $\mathcal{A}$ coincides with $G$. A natural way to show this is via Tits' Lemma, cf. Section 2.5.3, once one knows a geometry with $G$ as a sufficiently transitive group of automorphisms such that $\mathcal{A}$ is related to the amalgam of maximal parabolics induced by the action of $G$.

Such a geometry $\mathcal{G}_{A_{n}}$ has been identified in $[13,16,47]$ to be an $(n+1)$-dimensional non-degenerate unitary space $V$ over $\mathbb{F}_{q^{2}}$. The elements of $\mathcal{G}_{A_{n}}$ are the non-trivial proper non-degenerate subspaces $U$ of $V$, the type of a space $U$ being its dimension, incidence being defined by symmetrised containment. Using standard terminology from incidence geometry, one-dimensional elements of $\mathcal{G}_{A_{n}}$ are called points, two-dimensional elements lines. Fixing an orthonormal basis $e_{1}, \ldots, e_{n+1}$ of $V$, we consider the action of $G$ as a matrix group on $\mathcal{G}_{A_{n}}$ with respect to that basis. By Witt's Theorem, see [115], this action is flagtransitive, so that we can choose an arbitrary flag $F$ in order to describe the amalgam of parabolics.

This amalgam $\mathcal{A}\left(\mathcal{G}_{A_{n}}, G, F\right)$ of parabolics, cf. Section 2.5.2, turns out to have the same universal enveloping group as the amalgam $\mathcal{A}$ consisting of the blockdiagonal subgroups $\mathrm{SU}_{2}\left(q^{2}\right)$ and $\mathrm{SU}_{3}\left(q^{2}\right)$ and $\mathrm{SU}_{2}\left(q^{2}\right) \times \mathrm{SU}_{2}\left(q^{2}\right)$ of $G$ by the Redundancy Theorem from Section 2.5.4 and by [61, Lemma 29.3].

\subsubsection{Decomposing cycles}

The crucial observation for applying Tits' Lemma (Section 2.5.3) and the Redundancy Theorem (Section 2.5.4) is that $\mathcal{G}_{A_{n}}$ is almost always simply connected and has many simply connected residues. In [21] this simple connectedness is shown by proving that every cycle of the flag complex of $\mathcal{G}_{A_{n}}$ is null-homotopic, while in [47] it is proved in odd characteristic by studying certain subgroup complexes of $\mathrm{SU}_{n+1}\left(q^{2}\right)$. 
In this survey I will sketch the proof given in [21]. Fixing the base element $x$ to be a point, a standard technique based on residual connectedness allows to reduce every cycle of $\mathcal{G}_{A_{n}}$ to a cycle in the point-line incidence graph, i.e., the graph on the elements of dimension one and two with incidence as adjacency. Furthermore, every cycle in the point-line incidence graph can be understood as a cycle in the collinearity graph $\Gamma$ of $\mathcal{G}_{A_{n}}$, i.e., the graph consisting of the points of $\mathcal{G}_{A_{n}}$ as vertices in which two vertices are adjacent if and only if they lie on a common line of $\mathcal{G}_{A_{n}}$. A cycle in $\Gamma$ that is contained entirely within the residue of an element of $\mathcal{G}_{A_{n}}$ is called geometric and, being contained in a cone, is null-homotopic. Thus, simple connectedness of $\mathcal{G}_{A_{n}}$ follows, if one can prove that every cycle in $\Gamma$ can be decomposed into a product of geometric cycles.

A key fact exploited in [21] is that up to a few exceptions $\Gamma$ has diameter two. This implies that every cycle in $\Gamma$ is a product of cycles of length up to five and, thus, it suffices to show that every cycle of length three, four, and five is null-homotopic. When the dimension is large, one can always find a point that is perpendicular to all points on a fixed cycle, producing a decomposition of that cycle into geometric triangles. Hence proving simple connectedness is more or less trivial for large dimension. The difficulty of the proof lies in the case of small dimension, where [21] resorts to a case-by-case analysis.

To give the precise statement, let $n \geq 3$ and let $q$ be any prime power. Then the geometry $\mathcal{G}_{A_{n}}$ is simply connected, if $(n, q)$ is not one of $(3,2)$ and $(3,3)$. Since neither of these exceptions is simply connected, cf. Section 3.2.5, the result in [21] is optimal.

\subsubsection{Phan-type theorem of type $A_{n}$}

Altogether the Phan-type theorem of type $A_{n}$ follows:

Phan-type Theorem 1 (Bennett, Shpectorov [21]). Let $q$ be a prime power, let $n \geq 3$, and let $G$ be a group admitting a weak Phan system of type $A_{n}$ over $\mathbb{F}_{q^{2}}$.

(i) If $q \geq 4$, then $G$ is isomorphic to a central quotient of $\mathrm{SU}_{n+1}\left(q^{2}\right)$.

(ii) If $q=2,3$ and $n \geq 4$ and if, furthermore,

(a) for any triple $i, j, k$ of nodes of the Dynkin diagram $A_{n}$ that form a subdiagram

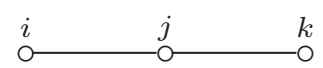

of type $A_{3}$, the subgroup $\left\langle U_{i, j}, U_{j, k}\right\rangle$ is isomorphic to a central quotient of $\mathrm{SU}_{4}\left(q^{2}\right)$;

(b) in case $q=2$ 


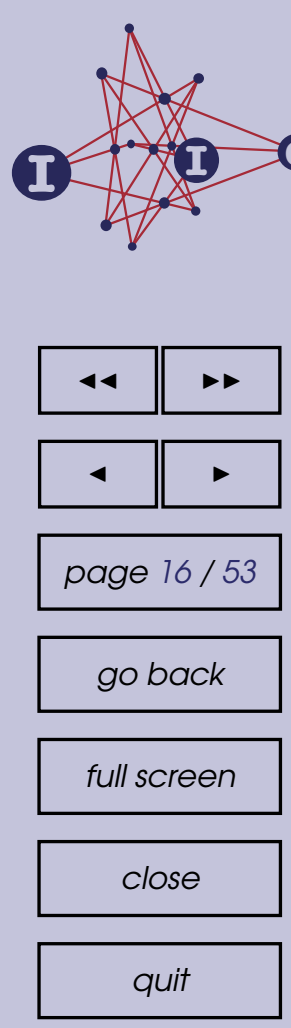

- for any triple $i, j, k$ of nodes of the Dynkin diagram $A_{n}$ that form a subdiagram

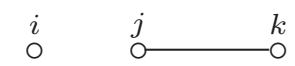

of type $A_{1} \oplus A_{2}$ the groups $U_{i}$ and $U_{j, k}$ commute elementwise; and - for any quadruple $i, j, k, l$ of nodes of the Dynkin diagram $\Delta$ that form a subdiagram

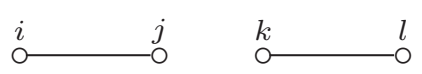

of type $A_{2} \oplus A_{2}$ the groups $U_{i, j}$ and $U_{k, l}$ commute elementwise; then $G$ is isomorphic to a central quotient of $\mathrm{SU}_{n+1}\left(q^{2}\right)$.

\subsubsection{The group $\mathrm{SU}_{4}\left(3^{2}\right)$}

The extra conditions in Phan-type Theorem 1 (ii) are due to the fact that for small $q$ and $n$ the geometry $\mathcal{G}_{A_{n}}$ is not simply connected. For example, [86] describes a group $H$ admitting a weak Phan system of type $A_{3}$ over $\mathbb{F}_{3^{2}}$ that is isomorphic to a non-split central extension of $\mathrm{SU}_{4}\left(3^{2}\right)$ by a group $K \cong(\mathbb{Z} / 3 \mathbb{Z})^{2}$, i.e. the sequence $1 \rightarrow K \rightarrow H \rightarrow \mathrm{SU}_{4}\left(3^{2}\right) \rightarrow 1$ is exact and non-split; in fact, $H$ is isomorphic to the Schur cover of $\mathrm{SU}_{4}\left(3^{2}\right)$. From there it is deduced in [86] that the geometry $\mathcal{G}_{A_{3}}$ admits a 9 -fold universal cover in case $q=3$.

\section{The Curtis-Tits Theorem}

Phan's theorems can be considered as a twisted version of the Curtis-Tits Theorem. Therefore by explaining the general setup of Phan-type theorems one naturally also describes a setup of the Curtis-Tits Theorem. In this section I will give many different (sometimes inequivalent) ways how to state the Curtis-Tits Theorem. Some versions deal with determining a Chevalley group (or even a Kac-Moody group) as the universal enveloping group of a certain amalgam, others with characterisations of these groups from purely local data. One version is merely concerned with the simple connectedness of a suitable chamber system. Each version has its advantages and disadvantages. While it may be easier for the geometric group theorist to prove the simple connectedness of some complex, a local group theorist may prefer to apply a version requiring only knowledge about local data. The transition from the former point of view to the latter requires a certain amount of rigidity of the complex on which the 



\subsubsection{Redundancy of the Steinberg presentation}

The Curtis-Tits Theorem states that Steinberg's presentation of Chevalley groups in Section 4.1.1 is highly redundant and that the amalgam consisting of rank one and rank two subgroups with respect to a system of fundamental roots of a maximal torus of a Chevalley group suffices to present this Chevalley group, cf. [45, 134], [135, Theorem 13.32].

The following version of the Curtis-Tits Theorem refers directly to the Steinberg presentation.

Curtis-Tits Theorem Version 1 (Curtis [45, Corollary 1.8]). Let $\Sigma$ be an indecomposable root system of rank at least two, let $\Pi$ be a fundamental system of $\Sigma$, and let $\mathbb{F}$ be an arbitrary field with five distinct elements. Define $G$ to be the abstract group with generators $\left\{x_{r}(t) \mid r \in \Sigma, t \in \mathbb{F}\right\}$ and defining relations

$$
x_{r}(t) x_{r}(u)=x_{r}(t+u), r \in \Sigma, t, u \in \mathbb{F},
$$

and for independent roots $r, s$,

$$
\left[x_{r}(t), x_{s}(u)\right]=\prod_{h, k} x_{h r+k s}\left(C_{h k r s} t^{h} u^{k}\right),
$$

with $h, k>0, h r+k s \in \Sigma$ (if there are no such numbers, then $\left[x_{r}(t), x_{s}(u)\right]=1$ ), and structure constants $C_{h k r s} \in\{ \pm 1, \pm 2, \pm 3\}$.

Let $A=\bigcup A_{i j}$, where $A_{i j}$ is the set of all roots which are linear combinations of the fundamental roots $r_{i}, r_{j} \in \Pi$. Let $G^{*}$ be the abstract group with generators $\left\{x_{r}(t) \mid r \in \Sigma, t \in \mathbb{F}\right\}$ and defining relations (4.1), for $r \in A$, and (4.2) for independent roots $r$, s belonging to some $A_{i j}$.

Then the natural epimorphism $G^{*} \rightarrow G$ is an isomorphism.

A more compact formulation (albeit without a concrete presentation) can be found in [62, 134], [135, Theorem 13.32]. Generalisations and variations on the theme are contained in $[33,132]$.

Curtis-Tits Theorem Version 2 (Gorenstein, Lyons, Solomon [62], Tits [135]). Let $G$ be the universal version of a Chevalley group of (twisted) rank at least three with root system $\Sigma$, fundamental system $\Pi$, and root groups $X_{\alpha}, \alpha \in \Sigma$. For each $J \subseteq \Pi$ let $G_{J}$ be the subgroup of $G$ generated by all root subgroups $X_{\alpha}, \pm \alpha \in J$. Let $D$ be the set of all subsets of $\Pi$ with at most two elements. Then $G$ is the universal enveloping group of the amalgam $\left(G_{J}\right)_{J \in D}$.

To look at a concrete example, consider the case of the universal SteinbergChevalley group of type $A_{n}$, which is $G=\mathrm{SL}_{n+1}(\mathbb{F})$. With the usual choices of 


\subsection{Buildings and twin buildings}

\subsubsection{Towards a Curtis-Tits geometry}

One purpose of this survey is to point out similarities between the Curtis-Tits Theorem on one hand and Phan's theorems on the other hand by describing suitable geometries whose simple connectedness yields the respective grouptheoretic identification result via Tits' Lemma [137] (Section 2.5.3). These geometries can be constructed using the opposition relation of a building or a twin building, cf. [4, 98, 139]. I start with a description of the ideas of proof of the Curtis-Tits Theorem given in $[4,98]$, whose generality actually implies that result for any two-spherical diagram (i.e., each sub-diagram of cardinality at most two is spherical), except that one has to exclude some small cases covered by the original Curtis-Tits Theorem. These exceptions arise from exactly those rank two diagrams and fields for which the geometry opposite a chamber inside the corresponding Moufang polygon is not connected, see [6, 28]. Before I am able to properly explain this geometric approach to the Curtis-Tits Theorem, I need to introduce the concepts of a building, a twin building, and of the opposite geometry.

A Chevalley group $G$ acts on its natural geometry, called a building. Buildings have been developed by Tits in numerous articles since the mid-1950's. The standard reference are Tits' lecture notes [135]. Other references are [3, 29, $81,111,115,146]$. For Coxeter groups and root systems see [25, 48, 90].

\subsubsection{Coxeter systems}

For a Coxeter matrix $M=\left(m_{i j}\right)_{i, j \in I}$ over some finite set $I$, i.e., a symmetric $|I| \times|I|$-matrix over $\mathbb{N} \cup\{\infty\}$ whose diagonal entries equal to one and whose off-diagonal entries are greater than or equal to two, the Coxeter diagram of $M$ is the complete labelled graph with vertex set $I$ and labels $m_{i j}$ on the edge $\{i, j\}$. The cardinality $|I|$ is called the rank of the Coxeter diagram. Usually the edges with label 2 are erased, so that it is meaningful to talk about connected or disconnected Coxeter diagrams.

Let $(W, S)$ be the Coxeter system of type $M$, i.e., $S=\left\{s_{i} \mid i \in I\right\}$ is a set and $W=\left\langle S \mid\left(s_{i} s_{j}\right)^{m_{i j}}=1\right\rangle$ is the quotient of the free group generated by $S$ and subject to the relations given by the Coxeter matrix $M$. The Coxeter system is spherical, if $|W|<\infty$, and irreducible, if the Coxeter diagram is connected. Irreducible spherical Coxeter diagrams have been classified, cf. [44].

Using the Bourbaki notation, the irreducible spherical Coxeter systems of rank at least three fall into the families $A_{n}, B_{n}, C_{n}, D_{n}$ plus the exceptional diagrams $E_{6}, E_{7}, E_{8}, F_{4}, H_{3}, H_{4}$. If $\Delta$ is a Coxeter diagram of type $M$, a Coxeter 


\begin{abstract}
$1+\frac{1}{1}$
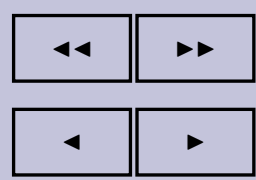

page $21 / 53$

system $(W, S)$ of type $M$ is also called a Coxeter system of type $\Delta$. For $J \subseteq I$, the pair $\left(W_{J}, S_{J}\right)$ consisting of $S_{J}=\left\{s_{i} \in S \mid i \in J\right\}$ and $W_{J}=\left\langle S_{J}\right\rangle$ is also a Coxeter system satisfying $W_{J}=\left\langle S_{J} \mid\left(s_{i} s_{j}\right)^{m_{i j}}=1\right\rangle$ by [25, Section IV.1.8, Theorem 2]. The group $W$ of a Coxter system $(W, S)$ is called a Coxeter group. It is in general not possible to reconstruct the Coxeter system from the abstract group $W$, see [18, 37, 40].
\end{abstract}

go back

full screen

close

quit

\title{
4.2.3. Buildings
}

A building of type $(W, S)$ (where $(W, S)$ is a Coxeter system) is a pair $\mathcal{B}=(\mathcal{C}, \delta)$ where $\mathcal{C}$ is a set and the distance function $\delta: \mathcal{C} \times \mathcal{C} \rightarrow W$ satisfies the following axioms for $x, y \in \mathcal{C}$ and $w=\delta(x, y)$.

(B1) $w=1$ if and only if $x=y$,

(B2) if $z \in \mathcal{C}$ is such that $\delta(y, z)=s \in S$, then $\delta(x, z)=w$ or $w s$; furthermore if $l(w s)=l(w)+1$, then $\delta(x, z)=w s$, and

(B3) if $s \in S$, there exists $z \in \mathcal{C}$ such that $\delta(y, z)=s$ and $\delta(x, z)=w s$.

The group $W$ is called the Weyl group of the building $\mathcal{B}$. The building $\mathcal{B}$ is called spherical, if its Weyl group $W$ is finite. Given a building $\mathcal{B}=(\mathcal{C}, \delta)$ one can define a chamber system on $\mathcal{C}$ in which two chambers $c$ and $d$ are $i$-adjacent, in symbols $c \sim_{i} d$, if and only if $\delta(c, d)=s_{i}$ or $\delta(c, d)=1$. The chamber system $\left(\mathcal{C},\left(\sim_{i}\right)_{i \in I}\right)$ uniquely determines $\mathcal{B}$, i.e., the $i$-adjacency relations on $\mathcal{C}$ determine the distance function $\delta$; cf. [136].

In this survey we only consider buildings $\mathcal{B}$ for which the chamber system $\mathcal{C}$ is thick. All thick spherical buildings with a connected Coxeter diagram $\Delta$ of rank at least three $(|\Delta|$ is also called the rank of the building) are known, e.g., by a local to global approach using the classification of Moufang buildings of rank two, see [141, Chapter 40], also [146]. This local to global approach is possible, because all thick spherical buildings of rank at least three with a connected Coxeter diagram are Moufang (see [135, Addendum]), whence their rank two residues are Moufang. Buildings of rank two are called generalised polygons, and are studied -Moufang or not- in [87, 104, 108, 143].

If $\mathcal{B}$ is a building, its chamber system contains a class of thin sub-chamber systems called apartments, each of which forms a building of the same type as $\mathcal{B}$. In an apartment $\Sigma$, for any $c \in \Sigma$ and $w \in W$, there is a unique chamber $d \in \Sigma$ such that $\delta(c, d)=w$. Every pair of chambers of $\mathcal{C}$ is contained in an apartment, cf. [146, Corollary 8.6]. The chamber system $\mathcal{C}$ defined by a building is always geometric; indeed buildings have the property, cf. Section 2.3.2, that for any set $\left\{\left(x_{i}, i\right) \mid i \in I\right\}$, with $x_{i}$ an $I \backslash\{i\}$-residue of $\mathcal{C}$, such that $x_{i} \cap x_{j} \neq \emptyset$ for all 

(iv) For any $w_{i} \in S$ we have $w_{i} B w_{i}^{-1} \neq B$;

(v) For any $w_{i} \in S$ and all $w \in W$ we have $w_{i} B w \subseteq(B w B) \cup\left(B w_{i} w B\right)$.

The pair of subgroups $B, N$ of $G$ is also called a $B N$-pair of $G$, see $[25,135]$. A group $G$ admitting a BN-pair satisfies

$$
G=\bigsqcup_{w \in W} B w B
$$

full screen

close

quit

For each $i \in I$ the set $P_{i}:=B \cup B w_{i} B$ is a subgroup of $G$. A Tits system $(G, B, N, S)$ leads to a building whose set of chambers equals $G / B$ and whose distance function

$$
\delta: G / B \times G / B \rightarrow W
$$

is given by $\delta(g B, h B)=w$ if and only if $B g^{-1} h B=B w B$. In the corresponding chamber system $g B$ and $h B$ are $i$-adjacent if and only if $B g^{-1} h B \subseteq B \cup B w_{i} B$.

\subsubsection{Twin buildings}

The simple connectedness of a building does not imply the Curtis-Tits Theorem, since the action of a Chevalley group on its building does not yield the correct amalgam. A class of geometries that yields the correct amalgams is best described using twin buildings. Twin buildings are obtained by relating two Tits buildings via a co-distance function, see $[2,3,101,113,114,140]$. Given two buildings $\mathcal{B}_{+}=\left(\mathcal{C}_{+}, \delta_{+}\right)$and $\mathcal{B}_{-}=\left(\mathcal{C}_{-}, \delta_{-}\right)$of the same type $(W, S)$, a co-distance, also called twinning, is a map

$$
\delta_{*}:\left(\mathcal{C}_{+} \times \mathcal{C}_{-}\right) \cup\left(\mathcal{C}_{-} \times \mathcal{C}_{+}\right) \rightarrow W
$$

such that the following axioms hold where $\epsilon= \pm, x \in \mathcal{C}_{\epsilon}, y \in \mathcal{C}_{-\epsilon}, w=\delta_{*}(x, y)$ :

(T1) $\delta_{*}(y, x)=w^{-1}$,

(T2) if $z \in \mathcal{C}_{-\epsilon}$ with $\delta_{-\epsilon}(y, z)=s \in S$ and $l(w s)=l(w)-1$, then $\delta_{*}(x, z)=w s$, and

(T3) if $s \in S$, there exists $z \in \mathcal{C}_{-\epsilon}$ such that $\delta_{-\epsilon}(y, z)=s \in S$ and $\delta_{*}(x, z)=w s$.

A twin building of type $(W, S)$ is a triple $\left(\mathcal{B}_{+}, \mathcal{B}_{-}, \delta_{*}\right)$, where $\mathcal{B}_{+}$and $\mathcal{B}_{-}$are buildings of type $(W, S)$ and $\delta_{*}$ is a twinning between $\mathcal{B}_{+}$and $\mathcal{B}_{-}$.

Every spherical twin building can be obtained in a unique way from some building $\mathcal{B}=(\mathcal{C}, \delta)$ of the same type $(W, S)$, cf. [140, Proposition 1]. Let $\mathcal{B}_{+}=$ $\left(\mathcal{C}_{+}, \delta_{+}\right)$be a copy of $\mathcal{B}$, let $\mathcal{B}_{-}=\left(\mathcal{C}_{-}, \delta_{-}\right)$be $\left(\mathcal{C}, w_{0} \delta w_{0}\right)$, and let $\delta_{*}$ be $w_{0} \delta$ 



\subsubsection{Examples of classical opposite geometries}

The following examples are descriptions of the opposite geometries for the four classical series of spherical buildings.

Example 1a. Let $\mathbb{F}$ be an arbitrary field and consider the universal SteinbergChevalley group $G \cong \mathrm{SL}_{n+1}(\mathbb{F})$ of type $A_{n}$ over $\mathbb{F}$. It corresponds to the building geometry $\mathcal{G}$ of type $A_{n}$, better known as the projective geometry, whose elements of type $i, 1 \leq i \leq n$, are the $i$-dimensional subspaces in an $(n+1)$-dimensional $\mathbb{F}$-vector space $V$. The geometries $\mathcal{G}_{+}$and $\mathcal{G}_{-}$are isomorphic to the projective geometry $\mathcal{G}$ and its dual, respectively. The latter is identical to $\mathcal{G}$ except that the types are interchanged by the map $i \mapsto n+1-i$. Elements $x_{+} \in \mathcal{G}_{+}$and $x_{-} \in \mathcal{G}_{-}$of type $i$ are opposite if they intersect trivially or, equivalently, form a direct sum decomposition $V=x_{+} \oplus x_{-}$, cf. [2, II, $\S 4$, Lemma 23]. These decompositions are the elements of $\mathcal{G}_{\mathrm{op}}$, where $x_{+} \oplus x_{-}$is incident to $x_{+}^{\prime} \oplus x_{-}^{\prime}$ if and only if $x_{\epsilon}$ is incident to $x_{\epsilon}^{\prime}$ for $\epsilon \in\{+,-\}$.

Example 2a. Let $G \cong \operatorname{Spin}_{2 n+1}(\mathbb{F})$ be the universal Steinberg-Chevalley group corresponding to the building geometry $\mathcal{G}$ of type $B_{n}$. The geometry $\mathcal{G}$ is the geometry of all totally isotropic subspaces of a non-degenerate $(2 n+1)$-dimensional orthogonal space $V$ over $\mathbb{F}$. In this case, both $\mathcal{G}_{+}$and $\mathcal{G}_{-}$are isomorphic to $\mathcal{G}$ and two $i$-dimensional totally isotropic subspaces $x_{+}$and $x_{-}$are opposite if $x_{-}$intersects the orthogonal complement of $x_{+}$trivially, i.e., $x_{+}^{\perp} \cap x_{-}=\{0\}$ or, equivalently, $x_{+}^{\perp} \oplus x_{-}=V$, [2, II, $\S 6$, Lemma 29]. Such pairs $\left(x_{+}, x_{-}\right)$are the elements of $\mathcal{G}_{\mathrm{op}}$, where $\left(x_{+}, x_{-}\right)$is incident to $\left(x_{+}^{\prime}, x_{-}^{\prime}\right)$ if and only if $x_{\epsilon}$ is incident to $x_{\epsilon}^{\prime}$ for $\epsilon \in\{+,-\}$.

Example 3a. Consider the universal Steinberg-Chevalley group $G \cong \mathrm{Sp}_{2 n}(\mathbb{F})$ of type $C_{n}$. In this case the corresponding building geometry $\mathcal{G}$ is the geometry of all totally isotropic subspaces of a non-degenerate $2 n$-dimensional symplectic space $V$ over $\mathbb{F}$. Both $\mathcal{G}_{+}$and $\mathcal{G}_{-}$are isomorphic to $\mathcal{G}$. Two $i$-dimensional totally isotropic subspaces $x_{+}$and $x_{-}$again are opposite if $x_{-}$intersects the orthogonal complement of $x_{+}$trivially, i.e., $x_{+}^{\perp} \cap x_{-}=\{0\}$ or, equivalently, $x_{+}^{\perp} \oplus x_{-}=V$, [2, II, $\S 6$, Lemma 29]. The pairs $\left(x_{+}, x_{-}\right)$are the elements of $\mathcal{G}_{\text {op }}$, where $\left(x_{+}, x_{-}\right)$ is incident to $\left(x_{+}^{\prime}, x_{-}^{\prime}\right)$ if and only if $x_{\epsilon}$ is incident to $x_{\epsilon}^{\prime}$ for $\epsilon \in\{+,-\}$.

Example 4a. Let $G \cong \operatorname{Spin}_{2 n}^{+}(\mathbb{F})$ be the universal Steinberg-Chevalley group of type $D_{n}$, to which corresponds the building geometry $\mathcal{G}$ of totally isotropic subspaces of a non-degenerate $2 n$-dimensional orthogonal space $V$ over $\mathbb{F}$ of Witt index $n$. In this case, both $\mathcal{G}_{+}$and $\mathcal{G}_{-}$are isomorphic to $\mathcal{G}$ up to interchanging the elements of types $n-1$ and $n$ in case $n$ odd. Two totally isotropic subspaces $x_{+}$and $x_{-}$of type $i$ are opposite, if $x_{-}$intersects the orthogonal complement of $x_{+}$trivially, i.e., $x_{+}^{\perp} \cap x_{-}=\{0\}$ or, equivalently, $x_{+}^{\perp} \oplus x_{-}=V$, [2, II, $\S 7$, 


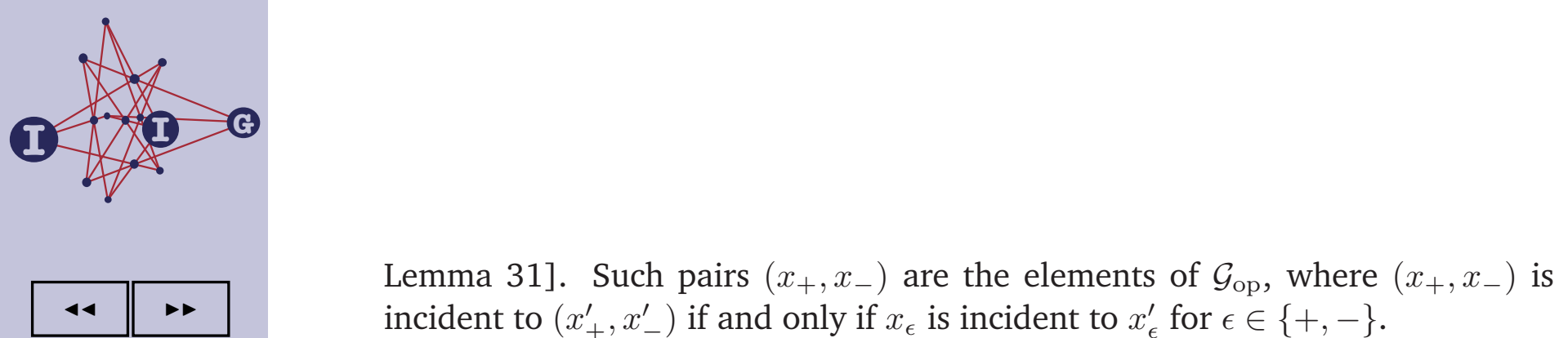
incident to $\left(x_{+}^{\prime}, x_{-}^{\prime}\right)$ if and only if $x_{\epsilon}$ is incident to $x_{\epsilon}^{\prime}$ for $\epsilon \in\{+,-\}$.

\subsubsection{The Curtis-Tits Theorem via geometric group theory}

If a twin building admits a strongly transitive group of automorphisms, i.e., a group acting transitively on the pairs of opposite chambers, the group acts flag-transitively on $\mathcal{G}_{\text {op }}$. In case the acting group is semisimple split algebraic or Kac-Moody, the stabilisers of the elements of a maximal flag of $\mathcal{G}_{\text {op }}$ are products of the type $G_{\Pi \backslash\{\alpha\}} Z(T)$, where $T$ is a maximal torus and $\Pi$ is a system of fundamental roots with respect to $T$. This setup together with Tits' Lemma (Section 2.5.3) and the Redundancy Theorem [78, Theorem 3.3] (Section 2.5.4) implies that the Curtis-Tits Theorem stated as in [62] (Section 4.1.2) follows from the following simple-connectedness result.

Curtis-Tits Theorem Version 4 (Abramenko, Mühlherr [4], Mühlherr [98]). Let $\mathcal{T}$ be a thick twin building with two-spherical diagram of rank at least three such that there is no rank two residue in $\mathcal{B}_{+}$or $\mathcal{B}_{-}$which is isomorphic to the buildings associated to $B_{2}(2), G_{2}(2), G_{2}(3)$ or ${ }^{2} F_{4}(2)$. Then $\operatorname{Opp}(\mathcal{T})$ is simply connected.

The proof of this theorem in [98] is derived directly from the axioms of twin buildings, properties of apartments in buildings, and certain connectedness properties of buildings like their simple connectedness. The exceptions in this approach come from the fact that the geometry opposite to a chamber in an arbitrary Moufang polygon is connected except in the cases $B_{2}(2), G_{2}(2), G_{2}(3)$ or ${ }^{2} F_{4}(2)$, cf. [6, 28]. Note in passing that in [2, II, $\S 2$, Proposition 9] it is shown that there is no hope for general connectedness results in the non-Moufang case. Of course, the Curtis-Tits Theorem for Steinberg-Chevalley groups does not have any exceptions by $[45,62,131,134,135]$.

In [4] the logic of proof is turned around. The authors prove the combinatorial Curtis-Tits Theorem Version 4 by directly proving the following generalisation of [135, Theorem 13.32]. The key is to construct an RGD system for $G$, cf. [140], also [109, 1.5], [3, Chapter 8].

Curtis-Tits Theorem Version 5 (Abramenko, Mühlherr [4], Mühlherr [98]). Let $\mathcal{T}$ be a thick twin building with two-spherical diagram $\Delta$ of rank at least three such that there is no rank two residue in $\mathcal{B}_{+}$or $\mathcal{B}_{-}$which is isomorphic to the buildings associated to $B_{2}(2), G_{2}(2), G_{2}(3)$ or ${ }^{2} F_{4}(2)$, let $G$ be a group acting transitively on the pairs of opposite chambers of $\mathcal{T}$, and let $\left(c_{+}, c_{-}\right)$be a pair of opposite chambers in $\mathcal{T}$. For each $J \subseteq \Delta$ let $G_{J}$ be the subgroup of $G$ stabilising the $J$-residue of $c_{+}$and the $J$-residue of $c_{-}$. Let $D$ be the set of all subsets of $\Delta$ with 

(F1) $\mathcal{C}_{+}^{\tau}=\mathcal{C}_{-}$,

(F2) $\tau$ flips the distances, i.e., $\delta_{\epsilon}(x, y)=\delta_{-\epsilon}\left(x^{\tau}, y^{\tau}\right)$ for $\epsilon= \pm$, and

(F3) $\tau$ preserves the co-distance, i.e., $\delta_{*}(x, y)=\delta_{*}\left(x^{\tau}, y^{\tau}\right)$

is called a flip. Notice that by (T1) of Section 4.2 .5 the element $\delta_{*}\left(x, x^{\tau}\right)$ is always an involution.

A flip satisfying the additional condition

(F4) there exists a chamber $c \in \mathcal{C}_{ \pm}$with $\delta_{*}\left(c, c^{\tau}\right)=1$

is called a Phan involution.

In case $\tau$ is a Phan involution the chamber system $\mathcal{C}_{\tau}$ whose chambers are pairs $\left(c, c^{\tau}\right)$ that belong to $\operatorname{Opp}(\mathcal{T})$, i.e.,

$$
\mathcal{C}_{\tau}=\left\{\left(c_{+}, c_{-}\right) \in \operatorname{Opp}(\mathcal{T}) \mid\left\{c_{+}, c_{-}\right\}=\left\{c_{+}^{\tau}, c_{-}^{\tau}\right\}\right\},
$$

is called the flipflop system of $\tau$. By (F4) the chamber system $\mathcal{C}_{\tau}$ is non-empty. By $[73,85]$ very many $\mathcal{C}_{\tau}$ are geometric, in particular all flipflop systems encountered in this survey. However, it is not known to me whether $\mathcal{C}_{\tau}$ is geometric in general. For a geometric flipflop system $\mathcal{C}_{\tau}$ denote by $\mathcal{G}_{\tau}$ the corresponding geometry, the flipflop geometry.

Following [52] one can alternatively define a Phan involution to be a flip of a twin building satisfying

(F4)' $\operatorname{proj}_{P} \tau \neq P$ for each panel $P$ of $\mathcal{T}$

where $\operatorname{proj}_{R} \tau:=\left\{x \in R \mid \operatorname{proj}_{R} \tau(x)=x\right\}$ for a spherical residue $R$ of $\mathcal{T}$ (Section 4.2.5). It is easily seen that a flip satisfying (F4)' also satisfies (F4). When talking about Phan involutions, we will generally only assume the validity of axioms (F1), (F2), (F3), (F4), unless explicitly stated otherwise.

\subsubsection{Flips of spherical twin buildings}

For a spherical twin building one can compute the action of $\tau$ on the Dynkin diagram of the building, see [66, Section 3.3]. Indeed, by Tits' characterisation each spherical twin building arises from a spherical building $\mathcal{B}=(\mathcal{C}, \delta)$ (cf. [140, Proposition 1] and also Section 4.2.5 of this survey) and we have

$$
\delta(c, d)=\delta_{+}(c, d)=\delta_{-}\left(c^{\tau}, d^{\tau}\right)=w_{0} \delta\left(c^{\tau}, d^{\tau}\right) w_{0}
$$





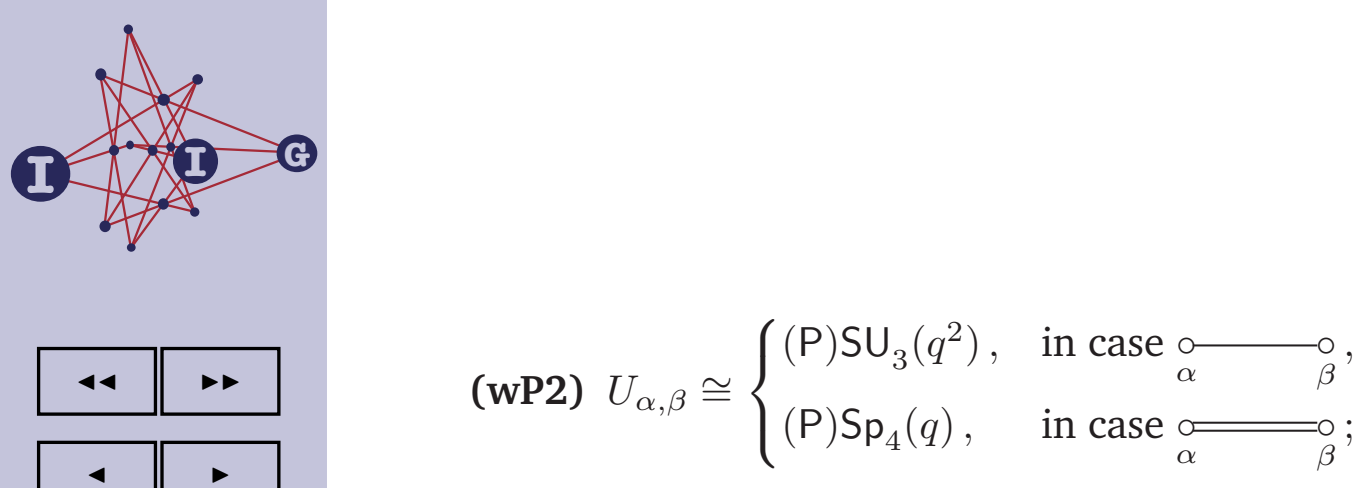

moreover, $U_{\alpha}$ and $U_{\beta}$ form a standard pair (see below) in $U_{\alpha, \beta}$, and

(wP3) $G=\left\langle U_{\alpha, \beta} \mid \alpha, \beta \in \Delta\right\rangle$.

For $U_{\alpha, \beta} \in\left\{\mathrm{SU}_{3}\left(q^{2}\right), \mathrm{Sp}_{4}(q)\right\}$ define $G_{\alpha, \beta}:=\mathrm{SL}_{3}\left(q^{2}\right), \mathrm{Sp}_{4}\left(q^{2}\right)$ accordingly. A standard pair in $U_{\alpha, \beta}$ is a pair of subgroups isomorphic to $\mathrm{SU}_{2}\left(q^{2}\right) \cong \mathrm{SL}_{2}(q)$ conjugate as a pair to the intersections $G_{\alpha} \cap U_{\alpha, \beta}$ and $G_{\beta} \cap U_{\alpha, \beta}$, where $G_{\alpha}, G_{\beta}$ form a pair of fundamental rank one subgroups of $G_{\alpha, \beta}$ (Section 4.1.2). Standard pairs in central quotients are defined as the images under the canonical homomorphism of standard pairs of the simply connected group. A concrete description of standard pairs of $\mathrm{SU}_{3}\left(q^{2}\right)$ can be found in Section 3.2.1. For a concrete description of standard pairs of $\mathrm{Sp}_{4}(q)$ see $[72,74]$.

\subsubsection{Phan-type theorem of type $B_{n}$}

The analogue of Aschbacher's geometry can be constructed from Example 2a (Section 4.3.2) as Example 1b (Section 5.1.1) has been deduced from Example 1a (Section 4.3.2).

Example 2b. Consider the situation of Example 2a, but with $\mathbb{F}=\mathbb{F}_{q^{2}}$, let $G \cong \Omega_{2 n+1}\left(q^{2}\right)$, i.e. the commutator subgroup of $\mathrm{GO}_{2 n+1}\left(q^{2}\right)$, and denote the form on $V$ by $(\cdot, \cdot)$. Since the case of even $q$ will be covered in Section 5.2.4 via the isomorphism $\operatorname{Spin}_{2 n+1}\left(2^{e}\right) \cong \operatorname{Sp}_{2 n}\left(2^{e}\right)$, it suffices to study the case of $q$ odd. Let $\left\{e_{1}, \ldots, e_{n}, f_{1}, \ldots, f_{n}, x\right\}$ be a hyperbolic basis of the orthogonal space $V$, so that $\left(e_{i}, f_{j}\right)=\delta_{i j}$, while $x$ with $(x, x)=1$ is orthogonal to each basis vector except itself. Consider the semilinear transformation $\tau$ of $V$ which is the composition of the linear transformation given by the Gram matrix of $(\cdot, \cdot)$ with respect to the above basis and the involutory field automorphism applied to the coordinates.

It can be shown, cf. [20], that $\tau$ produces a Phan involution of $\mathcal{T}$. Furthermore, $\mathcal{C}_{\tau}$ is geometric and $G_{\tau} \cong \mathrm{SO}_{2 n}(q)$ (cf. [20, Proposition 2.10]) acts flag-transitively on the corresponding flipflop geometry $\mathcal{G}_{\tau}$. The geometry $\mathcal{G}_{\tau}$ can be described as follows. For $u, v \in V$ let $((u, v))=\left(u, v^{\tau}\right)$. Then $((\cdot, \cdot))$ is a non-degenerate hermitian form. The flipflop geometry $\mathcal{G}_{\tau}$ can be identified via $\left(x_{+}, x_{-}\right) \mapsto x_{+}$with the geometry $\mathcal{G}_{B_{n}}$ of all subspaces of $V$ which are totally isotropic with respect to $(\cdot, \cdot)$ and, at the same time, non-degenerate with respect to $((\cdot, \cdot))$. 








\subsection{Phan's third theorem and the Phan-type theorem of type $E_{n}$}

\subsubsection{Phan's Theorem}

The article [107] also contains a theorem concerning the diagrams $E_{6}, E_{7}$, and $E_{8}$. Phan's Theorem 2 (Section 5.2.5) plus [106] are used in order to construct a system of subgroups satisfying the hypotheses of the Curtis-Tits Theorem Version 3, which then is invoked.

Phan's Theorem 3 (Phan [107]). Let $q \geq 5$ be odd. If $G$ admits a Phan system of type $E_{6}, E_{7}$, or $E_{8}$ over $\mathbb{F}_{q^{2}}$, then $G$ is isomorphic to a factor group of the universal Chevalley group ${ }^{2} E_{6}\left(q^{2}\right), E_{7}(q)$, or $E_{8}(q)$, respectively.

\subsubsection{Exploiting the filtrations}

By the Solomon-Tits-type Theorem 1 (Section 5.4.2) a generalised flipflop geometry of type $A_{3}$ over $\mathbb{F}_{q^{2}}$ is simply connected, provided $2^{2}(q+1)<q^{2}$, which is the case for $q \geq 5$, while a generalised flipflop geometry of type $A_{2}$ over $\mathbb{F}_{q^{2}}$ is connected, if $2(q+1)<q^{2}$, which is the case for $q \geq 3$. Together with the criterion for simple connectedness of a flipflop system from [52] (Section 5.3.3) this implies that the flipflop geometries of type $E_{6}, E_{7}, E_{8}$ over $\mathbb{F}_{q^{2}}$ are simply connected provided $q \geq 5$.

For completeness I should point out here that the chamber systems

$$
\left(A_{\tau}(R),\left(\sim_{t}\right)_{t \in J}\right)
$$

for residues $J$ of type $A_{1} \oplus A_{1}, A_{1} \oplus A_{2}, A_{1} \oplus A_{1} \oplus A_{1}$ are automatically (simply) connected by the following standard argument. Assuming that $\mathcal{G}=\mathcal{G}_{1} \oplus \mathcal{G}_{2}$ with $\mathcal{G}_{1}$ connected of rank at least two and $\mathcal{G}_{2}$ non-empty, the geometry $\mathcal{G}$ is simply connected. Indeed, the geometry $\mathcal{G}$ is certainly connected, and choosing a base point $x \in \mathcal{G}_{1}$ one can prove that any cycle originating at $x$ is homotopic to a cycle fully contained in $\mathcal{G}_{1}$. Such a cycle then is null homotopic because it forms a cone together with any element $z \in \mathcal{G}_{2}$.

\subsubsection{The Phan-type theorem of type $E_{n}$}

Alternatively —and this had already been done by Hoffman, Mühlherr, Shpectorov and the author roughly one year before the Solomon-Tits-type Theorem 1 was proved- one can directly compute the fundamental group for generalised flipflop geometries of type $A_{3}$. It turns out that via direct computation it is possible to show that the fundamental groups are trivial for $q \geq 4$. Together with the classification of amalgams [21,54] and the criterion for simple connectedness 


\begin{abstract}
$1+\frac{1}{1}$
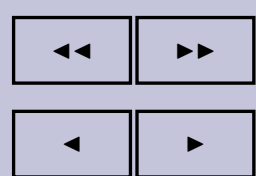

page $40 / 53$

of a flipflop system in [52] (Section 5.3.3) this implies the following Phan-type theorem.

Phan-type Theorem 5 (Gramlich, Hoffman, Mühlherr, Shpectorov 2005). Let $q \geq 4$ be a prime power and let $G$ be a group containing a weak Phan system of type $E_{6}, E_{7}$, or $E_{8}$ over $\mathbb{F}_{q^{2}}$. Then $G$ is isomorphic to a group of type ${ }^{2} E_{6}\left(q^{2}\right)$, $E_{7}(q)$, or $E_{8}(q)$.
\end{abstract}

go back

full screen

close

quit

\title{
5.6. The Abramenko filtration and the Phan-type theorem of type $F_{4}$
}

\subsubsection{Generalised flipflop geometries of type $B_{n}$ and $C_{n}$}

The criterion from Section 5.3.3 allows three-spherical diagrams. In view of the method of proof of the Phan-type theorem of type $E_{n}$ via generalised flipflop geometries of type $A_{3}$ it is natural to ask for the definition of generalised flipflop geometries of type $B_{3}$ and $C_{3}$.

Let $V$ be a vector space over a field $\mathbb{F}$ with an involution $\sigma$. For $U, W \leq V$, we say that $U$ is transversal to $W$ and write $U \pitchfork W$, if $U \cap W=0$ or $\langle U, W\rangle=V$. Note that $U \pitchfork W$ if and only if $\operatorname{dim}(U \cap W)=\max \{0, \operatorname{dim} U+\operatorname{dim} W-\operatorname{dim} V\}$. For a flag $F=\left(0=V_{0} \leq \ldots \leq V_{k}=V\right)$ and a subspace $U \leq V$ we say that $U$ is transversal to $F$ and write $U \pitchfork F$, if $U \pitchfork V_{i}$ for $0 \leq i \leq k$. This is the case if and only if $\left\langle U, V_{k_{U}}\right\rangle=V$ where $k_{U}=\min \left\{i \mid U \cap V_{j} \neq\{0\}\right\}$.

Given a flag $F=\left(0=V_{0} \leq \cdots \leq V_{k}=V\right)$ we call a family $\left(\omega_{i}\right)_{1 \leq i \leq k}$ of $\sigma$-hermitian forms $\omega_{i}: V_{i} \times V_{i} \rightarrow \mathbb{F}$ compatible with $F$ if $\operatorname{Rad}\left(\omega_{i}\right)=V_{i-1}$.

Let $F$ be as above and let $\omega=\left(\omega_{i}\right)_{i}$ be a family of compatible $\sigma$-hermitian forms. For $U \leq V$ we say that $U$ is transversal to $(F, \omega)$, if $U$ is transversal to $F$ and $U \cap V_{k_{U}}$ is $\omega_{k_{U}}$-non-degenerate. In this case we write $U \pitchfork(F, \omega)$.

Let $\Delta$ be the building geometry of type $B_{n}(\mathbb{F})$ or $C_{n}(\mathbb{F})$ embedded in a $\mathbb{F}$ vector space $V$ of dimension $2 n+1$, resp. $2 n$, and let $e_{1}, \ldots, e_{n}, f_{1}, \ldots, f_{n}, x$ be a standard hyperbolic basis of $V$ (where the vector $x$, of course, only occurs in case $\left.B_{n}\right)$. Let $F=\left(0=V_{0} \leq \cdots \leq V_{k}=V\right)$ be a flag satisfying $F^{\perp}=F$. Let $\omega$ be a family of $\sigma$-hermitian forms compatible with $F$ and assume that there is an $\omega_{k}$-non-isotropic vector that is $(\cdot, \cdot)$-isotropic. The generalised flipflop geometry of type $\Delta$ over $\mathbb{F}$ defined by $(F, \omega)$ consists of all subspaces $U$ of $V$ that are totally $(\cdot, \cdot)$-isotropic and transversal to $(F, \omega)$.

A closer look reveals that half of the forms $\omega_{i}$ actually do not play any role, because a totally isotropic subspace $U$ that is transversal to $F$ cannot meet any of the $V_{i}$ with $\operatorname{dim} V_{i} \leq n$. However, taking this into account would not simplify 


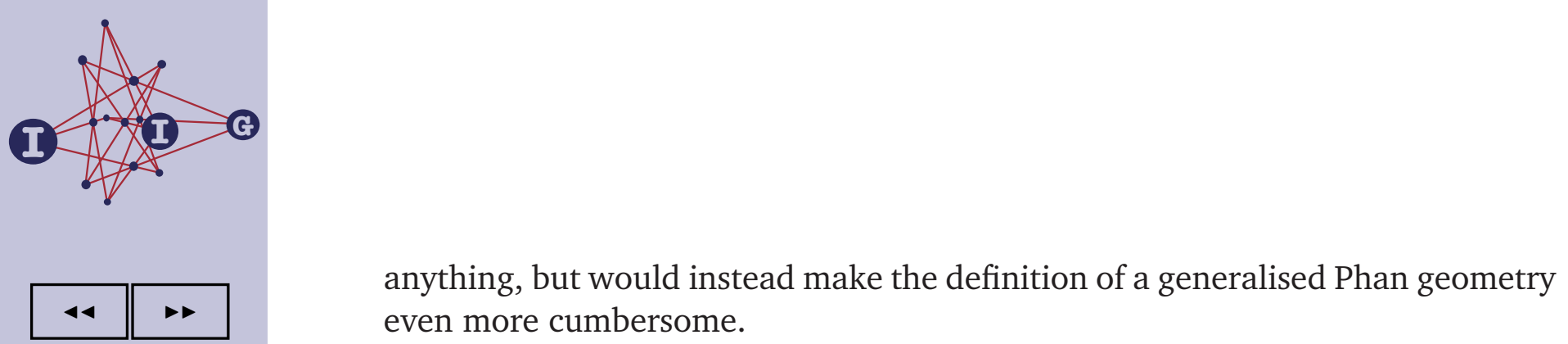
even more cumbersome.

\subsubsection{Another Solomon-Tits-type theorem and the Phan-type theorem of type $F_{4}$}

The concept of transversality introducted in Section 5.6.1 makes the Abramenko filtration from [2] accessible. This filtration has been used in [79] in order to prove the following theorem.

Solomon-Tits-type Theorem 2 (Gramlich, Witzel [79]). Let $\mathbb{F}$ be a field with an involution $\sigma$, let $\left(\mathcal{G}_{j}\right)_{1 \leq j \leq m}$ be a finite family of generalised flipflop geometries of type $B_{n}$ or $C_{n}$ embedded in some $(2 n+1)$ - or $2 n$-dimensional $\mathbb{F}$-vector space $V$, and let $\mathcal{G}=\bigcap_{j} \mathcal{G}_{j}$. In case $\mathbb{F}=\mathbb{F}_{q^{2}}$ assume $4^{n-1}(q+1) m<q^{2}$. Then $|\mathcal{G}|$ is homotopy equivalent to a wedge of $(n-1)$-spheres.

Similar to the case $A_{3}$, Hoffman, Mühlherr, Shpectorov and the author proved by direct computation that generalised flipflop geometries of type $B_{3}$ or $C_{3}$ are simply connected provided the underlying field contains at least 13 elements. Again using the simple connectedness criterion from [52] (Section 5.3.3), the final Phan-type theorem follows. Note that the generalised flipflop geometries are the correct objects in order to describe the chamber system $\left(A_{\tau}(R),\left(\sim_{t}\right)_{t \in J}\right)$ by [79, Proposition 6.6].

Phan-type Theorem 6 (Gramlich, Hoffman, Mühlherr, Shpectorov 2007). Let $q \geq 13$ be a prime power and let $G$ be a group containing a weak Phan system of type $F_{4}$ over $\mathbb{F}_{q^{2}}$. Then $G$ is isomorphic to a group of type $F_{4}(q)$.

It remains to study the cases of small $q$.

\section{Statement of the Phan-type theorem over finite fields}

We have reached one of the main purposes of this survey, the statement of the Phan-type theorem over finite fields. From Section 5.1.3 we know for which groups of Lie type the Phan-type theorem can make a statement, namely ${ }^{2} A_{n}$, $B_{n}, C_{n}, D_{2 n},{ }^{2} D_{2 n+1},{ }^{2} E_{6}, E_{7}, E_{8}, F_{4}$.

The Phan-type theorem for finite fields. Let $q \geq 3$, let $\Delta$ be a spherical Dynkin diagram of rank at least three, and let $G$ be a group with a weak Phan system of type $\Delta$ over $\mathbb{F}_{q^{2}}$. Then $G$ is isomorphic to a quotient of 


[3] P. Abramenko and K. S. Brown, Buildings - Theory and Applications, Grad. Texts in Math., vol. 248, Springer, Berlin, 2008.

[4] P. Abramenko and B. Mühlherr, Présentation de certaines $B N$-paires jumelées comme sommes amalgamées, C. R. Acd. Sci. Paris Sér. I Math. 325 (1997), 701-706.

[5] P. Abramenko and M. A. Ronan, A characterization of twin buildings by twin apartments, Geom. Dedicata 73 (1998), 1-9.

[6] P. Abramenko and H. Van Maldeghem, Connectedness of opposite-flag geometries in Moufang polygons, European J. Combin. 20 (1999), 461468.

[7] On opposition in spherical buildings and twin buildings, Ann. Comb. 4 (2000), 125-137.

[8] _ 1-twinnings of buildings, Math. Z. 238 (2001), 187-203.

[9] Characterisations of generalized polygons and opposition in rank 2 twin buildings, J. Geom. 74 (2002), 7-28.

[10] K. Altmann, The geometry of nondegenerate subspaces of complex orthogonal space, Master's thesis, TU Darmstadt, 2003.

[11] K. Altmann and R. Gramlich, On the hyperbolic unitary geometry, J. Algebraic Combin., to appear.

[12] _ On the geometry on the nondegenerate subspaces of nondegenerate orthogonal space, Bull. Belg. Math. Soc. Simon Stevin 13 (2006), 167-179.

[13] M. Aschbacher, Notes on the Curtis-Tits-Phan theorems, unpublished note.

[14] A characterization of Chevalley groups over fields of odd order, parts I, II, Ann. of Math. 106 (1977), 353-468.

[15] _ Correction to: "A characterization of Chevalley groups over fields of odd order, parts I, II", Ann. of Math. 111 (1980), 411-414.

[16] _ Simple connectivity of p-group complexes, Israel J. Math. 82 (1993), 1-43.

[17] Amer. Math. Soc. 51 (2004), 736-740. 
[18] P. Bahls, The isomorphism problem in Coxeter groups, Imperial College Press, London, 2005.

[19] C. D. Bennett, R. Gramlich, C. Hoffman and S. Shpectorov, CurtisPhan-Tits theory, in Groups, Combinatorics and Geometry: Durham 2001 (eds. A. A. Ivanov, M. W. Liebeck and J. Saxl), World Scientific, New Jersey, 2003, pp. 13-29.

[20] _ Odd-dimensional orthogonal groups as amalgams of unitary groups, part 1: general simple connectedness, J. Algebra 312 (2007), 426-444.

[21] C. D. Bennett and S. Shpectorov, A new proof of a theorem of Phan, $J$. Group Theory 7 (2004), 287-310.

[22] D. J. Benson, Representations and Cohomology. II: Cohomology of Groups and Modules, Cambridge University Press, Cambridge, 1991.

[23] R. J. Blok and C. Hoffman, A quasi Curtis-Tits-Phan theorem for the symplectic group, J. Algebra 319 (2008), 4662-4691.

[24] A Curtis-Tits-Phan theorem for the twin building of type $\tilde{A}_{n-1}$, J. Algebra 321 (2009), 1196-1224.

[25] N. Bourbaki, Lie groups and Lie algebras. Chapters 4-6, Elements of Mathematics (Berlin), Springer-Verlag, Berlin, 2002, translated from the 1968 French original by Andrew Pressley.

[26] G. E. Bredon, Topology and geometry, Grad. Texts in Math., vol. 139, Springer, Berlin, 1993.

[27] M. Bridson and A. Haefliger, Metric spaces of non-positive curvature, Springer, Berlin, 1999.

[28] A. E. Brouwer, The complement of a geometric hyperplane in a generalized polygon is usually connected, in Finite Geometries and Combinatorics (ed. F. D. C. et al.), 1993, pp. 53-57.

[29] K. S. Brown, Buildings, Springer, Berlin, 1989.

[30] F. Buekenhout (ed.), Handbook of incidence geometry, North-Holland, Amsterdam, 1995.

[31] F. Buekenhout and A. M. Cohen, Diagram geometry, Springer, Berlin, in preparation, http://www.win.tue.nl/ amc/buek. 
[32] F. Buekenhout and E. E. Shult, On the foundations of polar geometry, Geom. Dedicata 3 (1974), 155-170.

[33] P.-E. Caprace, On 2-spherical Kac-Moody groups and their central extensions, Forum Math. 19 (2007), 763-781.

[34] _ "Abstract" homomorphisms of split Kac-Moody groups, Mem. Amer. Math. Soc. 198 (2009).

[35] P.-E. Caprace and B. Mühlherr, Isomorphisms of Kac-Moody groups, Invent. Math. 161 (2005), 361-388.

[36] _ Isomorphisms of Kac-Moody groups which preserve bounded subgroups, Adv. Math. 206 (2006), 250-278.

[37] _ Reflection rigidity of 2-spherical Coxeter groups, Proc. Lond. Math. Soc. 94 (2007), 520-542.

[38] P.-E. Caprace and B. Rémy, Simplicité abstraite des groupes de KacMoody non affines, C. R. Math. Acad. Sci. Paris, Ser. I 342 (2006), 539544.

[39] , Simplicity and superrigidity of twin building lattices, Invent. Math. 176 (2009), 169-221.

[40] R. Charney and M. Davis, When is a Coxeter system determined by its Coxeter group?, J. Lond. Math. Soc. 61 (2000), 441-461.

[41] C. Chevalley, Certains schémas de groupes semi-simples, in $13 e$ Séminaire N. Bourbaki, 1960-1961, Benjamin, New York, 1966.

[42] A. M. Cohen, Point-line spaces related to buildings, in Buekenhout [30], pp. 647-737.

[43] A. M. Cohen, H. Cuypers and R. Gramlich, Local recognition of nonincident point-hyperplane pairs, Combinatorica 25 (2005), 271-296.

[44] H. S. Coxeter, The complete enumeration of finite groups of the form $R^{2}=\left(R_{i}, R_{j}\right)^{k_{i j}}=1$, J. Lond. Math. Soc. 10 (1935), 21-25.

[45] C. W. Curtis, Central extensions of groups of Lie type, J. Reine Angew. Math. 220 (1965), 174-185.

[46] H. Cuypers, Symplectic geometries, transvection groups, and modules, J. Combin. Theory Ser. A 65 (1994), 39-59.

[47] K. M. Das, Homotopy and homology of p-group complexes, Ph.D. thesis, Caltech (1994). 
[48] M. Davis, The geometry and topology of Coxeter groups, London Mathematical Society, 2008.

[49] A. Delgado, D. M. Goldschmidt and B. Stellmacher, Groups and graphs: new results and methods, DMV Seminar, vol. 6, Birkhäuser, Basel, 1985.

[50] M. Demazure, Schémas en groupes réductifs, Bull. Soc. Math. France 93 (1965), 369-413.

[51] A. Devillers, R. Gramlich and B. Mühlherr, The sphericity of the complex of nondegenerate subspaces, J. London Math. Soc. 79 (2009), 684700 .

[52] A. Devillers and B. Mühlherr, On the simple connectedness of certain subsets of buildings, Forum Math. 19 (2007), 955-970.

[53] A. Dress and R. Scharlau, Gated sets in metric spaces, Aequiationes Math. 34 (1987), 112-120.

[54] J. R. Dunlap, Uniqueness of Curits-Phan-Tits amalgams, Ph.D. thesis, Bowling Green State University (2005).

[55] B. Fischer, Finite groups generated by 3-transpositions, I, Invent. Math. 13 (1971), 232-246.

[56] H. Freudenthal, Oktaven, Ausnahmegruppen und Oktavengeometrie, Mathematisch Instituut der Rijksuniversiteit te Utrecht, Utrecht, 1951.

[57] _ Oktaven, Ausnahmegruppen und Oktavengeometrie, Geom. Dedicata 19 (1985), 7-63.

[58] H. Garland, $p$-adic curvature and the cohomology of discrete subgroups of $p$-adic groups, Ann. of Math. 97 (1973), 375-423.

[59] D. M. Goldschmidt, Automorphisms of trivalent graphs, Ann. of Math. 111 (1980), 377-406.

[60] D. Gorenstein, R. Lyons and R. Solomon, The classification of the finite simple groups, Math. Surveys Monographs, vol. 40.1, American Mathematical Society, Providence, 1994.

[61] - The classification of the finite simple groups. Number 2. Part I. Chapter G. General group theory, Math. Surveys Monogr., vol. 40.2, American Mathematical Society, Providence, 1995.

[62] - The classification of the finite simple groups. Number 3. Part I. Chapter A. Almost simple K-groups, Math. Surveys Monogr., vol. 40.3, American Mathematical Society, Providence, 1998. 

, The classification of the finite simple groups. Number 4. Part II. Chapters 1-4. Uniqueness Theorems, Math. Surveys Monogr., vol. 40.4, American Mathematical Society, Providence, 1999.

[64] , The classification of the finite simple groups. Number 5. Part III. Chapters 1-6. The generic case, stages 1-3a, Math. Surveys Monogr., vol. 40.5, American Mathematical Society, Providence, 2002.

[65] - The classification of the finite simple groups. Number 6. Part IV. The special odd case, Math. Surveys Monogr., vol. 40.6, American Mathematical Society, Providence, 2006.

[66] R. Gramlich, On graphs, geometries, and groups of Lie type, Ph.D. thesis, TU Eindhoven (2002), http://alexandria.tue.nl/extra2/200211439.pdf.

[67] _ Line-hyperline pairs of projective spaces and fundamental subgroups of linear groups, Adv. Geom. 4 (2004), 83-103.

[68] _ On the hyperbolic symplectic geometry, J. Combin. Theory Ser. A 105 (2004), 97-110.

[69] - Weak Phan systems of type $C_{n}$, J. Algebra 280 (2004), 1-19.

[70] R. Gramlich, J. I. Hall and A. Straub, The local recognition of reflection graphs of spherical Coxeter groups, J. Algebraic Combin., to appear.

[71] R. Gramlich, C. Hoffman, W. Nickel and S. Shpectorov, Evendimensional orthogonal groups as amalgams of unitary groups, J. Algebra 284 (2005), 141-173.

[72] R. Gramlich, C. Hoffman and S. Shpectorov, A Phan-type theorem for $\operatorname{Sp}(2 n, q)$, J. Algebra 264 (2003), 358-384.

[73] R. Gramlich, M. Horn and B. Mühlherr, Abstract involutions of algebraic groups and of Kac-Moody groups, submitted.

[74] R. Gramlich, M. Horn and W. Nickel, The complete Phan-type theorem for $\operatorname{Sp}(2 n, q)$, J. Group Theory 9 (2006), 603-626.

[75] _ Odd-dimensional orthogonal groups as amalgams of unitary groups, part 2: machine computations, J. Algebra 316 (2007), 591-607.

[76] R. Gramlich, M. Horn, A. Pasini and H. Van Maldeghem, Intransitive geometries and fused amalgams, J. Group Theory 11 (2008), 443-464. 
[77] R. Gramlich and B. Mühlherr, Lattices from involutions of Kac-Moody groups, Oberwolfach Rep. 5 (2008), 139-140.

[78] R. Gramlich and H. Van Maldeghem, Intransitive geometries, Proc. Lond. Math. Soc. 93 (2006), 666-692.

[79] R. Gramlich and S. Witzel, The sphericity of generalized Phan geometries of type $B_{n}$ and $C_{n}$ and the Phan-type theorem of type $F_{4}$, submitted.

[80] J. L. Gross and T. W. Tucker, Topological graph theory, Wiley, New York, 1987.

[81] T. Grundhöfer, Basics on buildings, in Tent [126], pp. 1-21.

[82] J. I. Hall, The hyperbolic lines of finite symplectic spaces, J. Combin. Theory Ser. A 47 (1988), 284-298.

[83] _ Graphs, geometry, 3-transpositions, and symplectic $\mathbb{F}_{2^{-}}$ transvection groups, Proc. London Math. Soc. 58 (1989), 89-111.

[84] M. Horn, Amalgams of unitary groups in $S p(2 n, q)$, Master's thesis, TU Darmstadt (2005).

[85] _ Involutions of Kac-Moody groups, Ph.D. thesis, TU Darmstadt (2008).

[86] On the Phan system of the Schur cover of SU $\left(4,3^{2}\right)$, Des. Codes Cryptogr. 47 (2008), 243-247.

[87] D. R. Hughes and F. C. Piper, Projective planes, Springer, Berlin, 1973.

[88] J. E. Humphreys, Remarks on "A theorem on special linear groups", J. Algebra 22 (1972), 316-318.

[89] _ The Steinberg representation, Bull. Amer. Math. Soc. (N.S.) 16 (1987), 247-263.

[90] _ Reflection groups and Coxeter groups, Cambridge University Press, Cambridge, 1990.

[91] A. A. Ivanov, Geometry of sporadic groups I: Petersen and tilde geometries, Encyclopedia Math. Appl., vol. 76, Cambridge University Press, 1999.

[92] A. A. Ivanov and S. Shpectorov, Geometry of sporadic groups II: representations and amalgams, Encyclopedia Math. Appl., vol. 91, Cambridge University Press, 2002. 
[93] F. Klein, Vergleichende Betrachtungen über neuere geometrische Forschungen, Verlag von Andreas Deichert, Erlangen, 1872, also known as Erlanger Programm.

[94] A. S. Küsefoglu, The second degree cohomology of finite orthogonal groups. I, J. Algebra 56 (1979), 207-220.

[95] _ The second degree cohomology of finite orthogonal groups. II, J. Algebra 67 (1980), 88-109.

[96] G. Margulis, Discrete subgroups of semisimple Lie groups, Springer, Berlin, 1991.

[97] B. Mühlherr, Some contributions to the theory of buildings based on the gate property, Ph.D. thesis, Uni Tübingen (1994).

[98] _ On the simple connectedness of a chamber system associated to a twin building (1996), unpublished note.

[99]_, A rank 2 characterization of twinnings, European J. Combin. 19 (1998), 603-612.

[100] L Locally split and locally finite twin buildings of 2-spherical type, J. Reine Angew. Math. 511 (1999), 119-143.

[101] , Twin buildings, in Tent [126], pp. 103-117.

[102] A. Pasini, Some remarks on covers and apartments, in Finite geometries (eds. C. A. Baker and L. M. Batten), Lect. Notes Pure Appl. Math., vol. 103, Dekker, New York, 1985, pp. 223-250.

[103] D Diagram geometries, Clarendon Press, Oxford, 1994.

[104] S. E. Payne and J. A. Thas, Finite generalized quadrangles, Pitman, Boston, 1984.

[105] K. Phan, A theorem on special linear groups, J. Algebra 16 (1970), 509518.

[106] _ On groups genererated by three-dimensional special unitary groups, I, J. Aust. Math. Soc. Ser. A 23 (1977), 67-77.

[107] _ On groups genererated by three-dimensional special unitary groups, II, J. Aust. Math. Soc. Ser. A 23 (1977), 129-146.

[108] G. Pickert, Projektive Ebenen, Springer, Berlin, 1975, second edition. 
[109] B. Rémy, Groupes de Kac-Moody déployés et presque déployés, Astérisque, vol. 277, Société Mathématiques de France, Paris, 2002.

[110] A. E. Roberts, A Phan-type theorem for orthogonal groups, Ph.D. thesis, Bowling Green State University (2005).

[111] M. A. Ronan, Lectures on buildings, Perspectives Math., vol. 7, Academic Press, Boston, 1989.

[112] _ Local isometries of twin buildings, Math. Z. 234 (2000), 435455.

[113] _ Twin trees and twin buildings, in Tent [126], pp. 119-137.

[114] M. A. Ronan and J. Tits, Twin trees. I., Invent. Math. 116 (1994), 463479.

[115] R. Scharlau, Buildings, in Buekenhout [30], pp. 477-645.

[116] H. Seifert and W. Threlfall, Lehrbuch der Topologie, Chelsea Publishing Company, New York, 1934.

[117] J.-P. Serre, Arbres, amalgames, $S L_{2}$, Astérisque, vol. 46, Soc. Math. France, Paris, 1977.

[118] _ Trees, Springer, Berlin, 2003, corrected second printing.

[119] L. Solomon, The Steinberg character of a finite group with $B N$-pair, in Theory of finite groups, Benjamin, New York, 1969, pp. 213-221.

[120] E. H. Spanier, Algebraic topology, McGraw-Hill, New York, 1966.

[121] R. Steinberg, Générateurs, relations, et revêtements de groupes algébriques, in Colloque sur la théorie des groupes algébriques, Bruxelles, 1962, pp. 113-127.

[122] _ Lectures on Chevalley groups, Mimeographed lecture notes, Yale University, New Haven (1968).

[123] M. Stroppel, Reconstruction of incidence geometries from groups of automorphisms, Arch. Math. 58 (1992), 621-624.

[124] A categorial glimpse at the reconstruction of geometries, Geom. Dedicata 46 (1993), 47-60.

[125] G. Stroth, Some geometries for McL, Comm. Algebra 17 (1989), 28252833. 


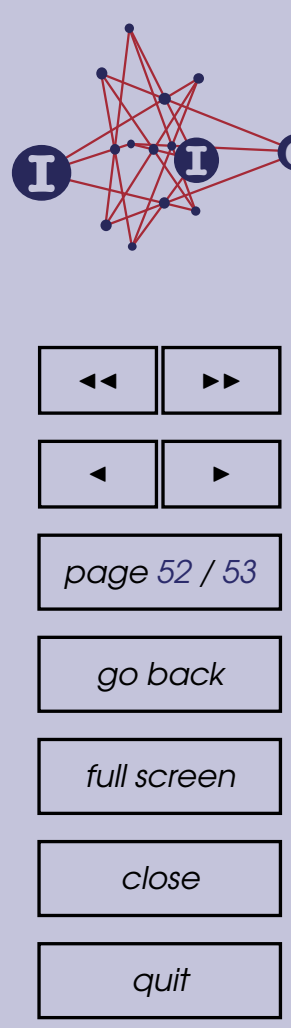

[126] K. Tent (ed.), BN-pairs and groups of finite Morley rank. Tits buildings and the model theory of groups (Würzburg, 2000), Cambridge University Press, Cambridge, 2002.

[127] F. G. Timmesfeld, Groups generated by k-root subgroups, Invent. Math. 106 (1991), 575-666.

[128] , Presentations for certain Chevalley groups, Geom. Dedicata 73 (1998), 85-117.

[129] _ Abstract root subgroups and quadratic action, Adv. Math. 142 (1999), 1-150.

[130] _ Abstract root subgroups and simple groups of Lie type, Monogr. Math., vol. 95, Birkhäuser, Basel, 2001.

[131] , On the Steinberg-presentation for Lie-type groups, Forum Math. 15 (2003), 645-663.

[132] _ , The Curtis-Tits presentation, Adv. Math. 189 (2004), 38-67.

[133] _ Steinberg-type presentations for Lie-type groups, J. Algebra 300 (2006), 806-819.

[134] J. Tits, Groupes semi-simples isotropes, in Colloque sur la théorie des groupes algébriques, Bruxelles, 1962, pp. 137-146.

[135] _ Buildings of spherical type and finite BN-pairs, Lecture Notes in Math., vol. 386, Springer, Berlin, 1974.

[136] , A local approach to buildings, in The Geometric Vein - The Coxeter Festschrift (eds. C. Davis, B. Grünbaum and F. A. Sherk), Springer, New York, 1981, pp. 519-547.

[137] _ Ordonnés, immeubles et sommes amalgamées, Bull. Soc. Math. Belg. Sér. A 38 (1986), 367-387.

[138] Uniqueness and presentation of Kac-Moody groups over fields, J. Algebra 105 (1987), 542-573.

[139] , Résumé de cours, in Annuaire du Collège de France, 89e année, 1989-1990, Collège de France, 1990, pp. 87-103.

[140] , Twin buildings and groups of Kac-Moody type, in Groups, Combinatorics and Geometry (eds. M. W. Liebeck and J. Saxl), London Math. Soc. Lecture Note Ser., vol. 165, Cambridge University Press, Cambridge, 1992, pp. 249-286. 
